\title{
Steganography Genetic Algorithm Hyperparameter Tuning through Response Surface Methodology
}

\author{
Warley Gramacho da Silva ${ }^{1}$, Rafael Lima de Carvalho ${ }^{1}$ and Glêndara Aparecida de Souza Martins ${ }^{2}$ \\ ${ }^{1}$ Federal University of Tocantins - UFT, Computer Science Department, Palmas-TO, Brazil \\ ${ }^{2}$ Federal University of Tocantins - UFT, Food Engineering Department, Palmas-TO, Brazil
}

Reception date of the manuscript: 27/02/2020

Acceptance date of the manuscript: 28/02/2020

Publication date: 09/03/2020

\begin{abstract}
Steganography consists of hidding bits of an information source into a host source. In image processing, a common way of doing the hiding process is to break each byte from the message information and embbed into the message bytes in a way that the differences among the original host and the embedded one are minimized. A Genetic Algorithm (GA) can be used to find the proper combination of bits in order to minimize such differences, but some hyperparameters need to be optimized in order to get an optimized performance. This work investigates the application of Response Surface Methodology (RSM) to find the best hyperparameters of a genetic algorithm applied to image steganography. As a result, RSM was able to point out fine-tuning hyperparameters for the GA.
\end{abstract}

Keywords—RSM, Steganography, Genetic Algorithms, Hyperparameter Optimization

\section{INTRODUÇÃO}

A esteganografia é a arte de ocultar informações digitais, a fim de impedir a detecção de mensagens ocultas, ou seja, o objetivo da esteganografia é evitar a suspeita na transmissão de uma mensagem secreta. Inclui-se técnicas para ocultar uma imagem, um arquivo de texto, um arquivo de áudio e até mesmo um programa executável dentro de uma imagem de cobertura sem distorção visual da imagem $[1,2]$. Como apresentado em [3], esteganografia pode ser formalmente definida como o processo de incorporação que descreve um mapeamento de $E: C \times M \rightarrow C$, em que $C$ é o conjunto de coberturas possíveis e $M$ é o conjunto de possíveis mensagens. O processo de extração consiste em um mapeamento de $D: C \rightarrow M$, extraindo a mensagem secreta de uma imagem de cobertura.

Definição I.1 $O$ quádruplo $\Psi=<C, M, D, E>$, onde $C$ é o conjunto de coberturas possíveis, $M$ o conjunto de mensagens secretas com $|C| \geq|M|, E: C \times M \rightarrow C$ a função de mapeamento e $D: C \rightarrow M$, a função de extração, com a propriedade que $D(E(c, m))=m$ para todos os $m \in M$ e $c \in C$ [3].

O processo de mapeamento é definido de forma que a imagem de cobertura e o objeto "stego" correspondente sejam perceptivamente semelhantes. O esquema de esteganografia pode ser visto na Figura 1.

Contact data: Warley Gramacho da Silva, 109 Norte, Av. NS15, Câmpus

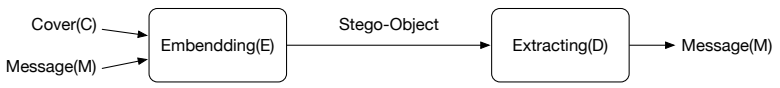

Fig. 1: Fluxograma de esteganografia

De acordo com [2], existem seis categorias de esteganografia, a saber: técnicas de sistema de substituição, técnicas de transformação de domínio, técnicas de espectro de espalhamento, técnicas de método estatístico, técnicas de distorção e técnicas de geração de cobertura. $\mathrm{Na}$ técnica do sistema de substituição, os bits redundantes ou desnecessários são subistituidos de uma imagem de cobertura pelos bits da mensagem secreta usando, por exemplo, o método Bit Menos Significativo (LSB, do inglês, Least Significant Bit) para codificar a mensagem secreta. No entanto, a esteganografia do LSB é suscetível as técnicas de análise de esteganização [4]. Para evitar a instalação de detecção de esteganografia através do uso do método LSB, em [5], foi proposta uma nova estratégia de substituição dos bits. Com o objetivo de otimizar o desempenho desse esquema proposto, foi utilizado um Algoritmo Genético (GA).

Os GAs possuem alguns parâmetros de entrada que são, geralmente, definidos manualmente após várias tentativas de ajustes. Uma técnica usada para definir um ajuste para os parâmetros dos AGs é a técnica da Metodologia da Superfície de Resposta (RSM). O RSM é uma combinação de técnicas matemáticas e estatísticas adequadas para modelagem e análise de problemas nos quais a variável resposta é afetada por várias variáveis de entrada, que visam otimizar as respostas [6].

Universitário de Palmas, wgramacho@uft.edu.br 


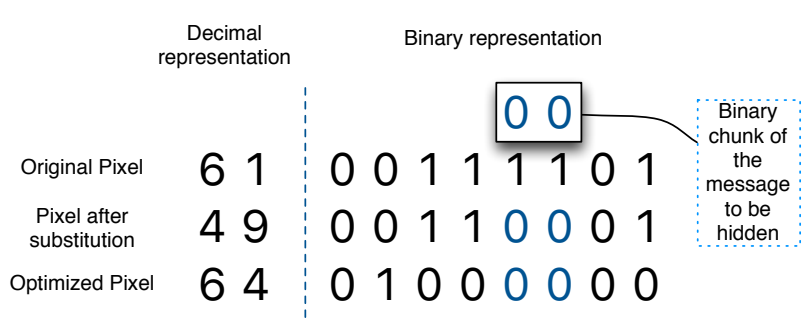

Fig. 2: Modelo de otimização adotado. Na imagem, o pixel original (valor 61 em decimal) recebe o par de pixels (00). Após a substituição, o valor do pixel otimizado se torna 64 .

Neste artigo, propõe-se o uso da MRS para melhor ajuste dos parâmetros do AG para otimizar a esteganografia de imagens usando uma estratégia de substituição dos bits conforme proposto por [5].

\section{Algoritmo Genético para o Prob- LEMA DE ESTEGANOGRAFIA}

Algoritmos Genético é uma metahaurística de otimização bio-inspirada, proposta por John Holland nos anos 1960 [7]. O GA é um método de busca e otimização que simula os processos naturais da evolução que consistem basicamente nos processos de cruzamento, mutação, mapeamento de fenótipo e calculo de aptidão (fitness) [8].

A idéia básica do GA é mostrada no Algoritmo 1 e funciona da seguinte maneira. Uma população inicial é gerada. Depois disso, o loop do algoritmo genético começa com a etapa de cruzamento, onde dois indivíduos são selecionados para serem os pais de um novo indivíduo, essa etapa é repetida até que não haja mais pais. Logo após a mutação ocorrer, é necessária uma baixa taxa de mutação nesta etapa para não comprometer a geração. E, finalmente, o processo de seleção natural. Para concluir o loop, um critério de parada é avaliado (número máximo de iteração).

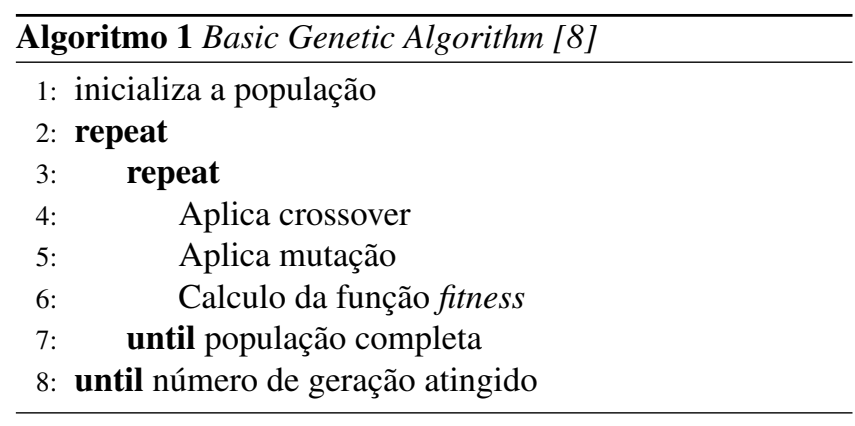

O princípio de otimização usado neste trabalho segue o proposto em [5]. Nesta abordagem, cada byte de destino deve passar por uma fase de verificação. Para ilustrar como isso funciona, considere um byte, como ilustrado na Figura 2. Na figura, o primeiro byte (61) é o valor original do pixel encontrado na imagem de destino. Neste exemplo, a mensagem será colocada na terceira e quarta posição do pixel de destino. Ao fazer a substituição, o valor do pixel se torna 49, o que fornece uma diferença de luminância de 12. Depois de otimizar o valor do pixel, pode ser visto que o valor 64 está mais próximo do valor original, enquanto o bloco de mensagens a ser oculto permanece incorporado.

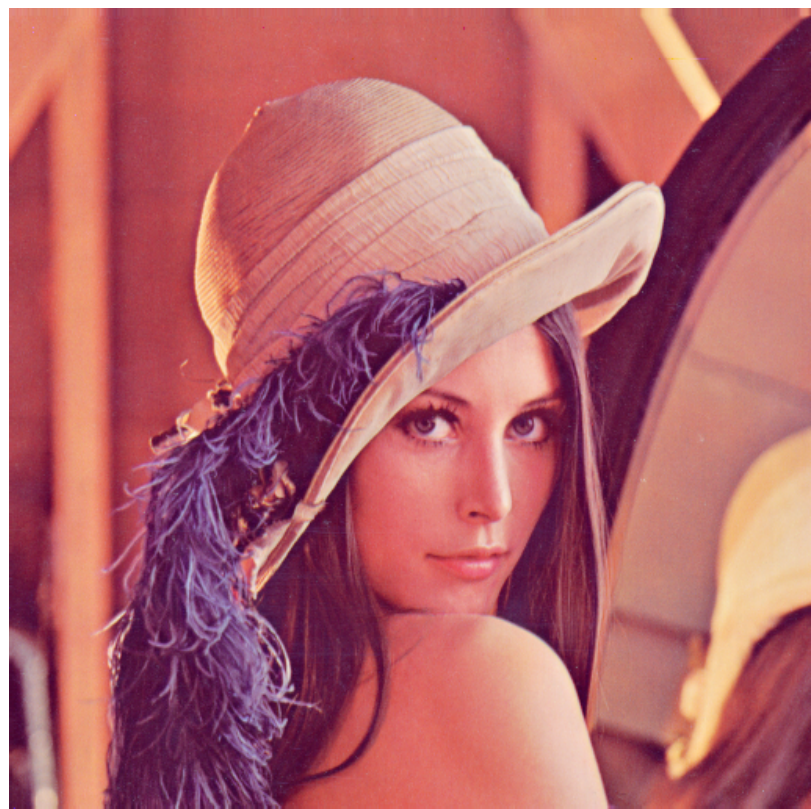

Fig. 3: Imagem-alvo utilizada nos experimentos.

A representação binária do pixel favorece uma escolha natural para abordagem de otimização tais como Algoritmos Genéticos. Neste artigo, a função de aptidão (fitness) é uma função quadrática que contabiliza a diferença entre os pixels originais e os otimizados. Esta função pode ser conforme.

$$
\sum_{i}^{N}\left(\text { pixel }_{i}-\text { optimizedPixel }_{i}\right)^{2}
$$

A solução ótima para o problema é a mais próxima do conjunto original de pixels, a população do Algoritmo Genético é gerada tendo pontos aleatórios ao redor da imagem original. Depois disso, a população inicial é atualizada para manter os bits da mensagem oculta. Em sequência, o fitness de cada individuo é calculado para prosseguir com o restante do algoritmo AG.

\section{EXPERIMENTOS}

Para execução do AG, a Figura 3 foi utilizada para realização dos testes juntamente com uma mensagem de texto aleatória a ser oculta na imagem de destino.

Para ilustrar a abordagem de ajuste dos parâmetros do AG, selecionou-se um conjunto de parâmetros para observação das respectivas influências no desempenho do AG, independentemente do problema estudado. Neste caso, os seguintes parâmetros foram analisados: probabilidade de mutação ( $p M u t)$, probabilidade de cruzamento ( $p C r o s)$, tamanho da população ( $s P o p)$ e número de gerações $(n G e n)$.

Empregou-se nesse projeto o planejamento fatorial completo $2^{k}$, com $k=4$ parâmetros do AG (fatores principais) e três níveis. Os níveis são mostrados como -1 , quando o fator está em um nível mínimo, 0 , quando o fator esta no nível central, e +1 , quando o fator pretendido está em um nível máximo [9]. Os parâmetros e seus níveis correspondentes podem ser vistos na Tabela 1 .

Os experimentos realizados têm por objetivo buscar o melhor ajuste dos parâmetros de entrada do Algoritmo Genético de tal modo que sejam encontradas as melhores respostas médias para a função fitness. Desta forma, foram executadas 
TABLE 1: VALORES PARA OS PARÂMETROS DO AG.

\begin{tabular}{cccccc}
\hline \hline Parâmetros do AG & Codificado & pCros & pMut & sPop & nGen \\
\hline \hline Mínimo & -1 & 0.01 & 0.01 & 10 & 10 \\
\hline Central & 0 & 0.50 & 0.50 & 105 & 105 \\
\hline Máximo & 1 & 0.99 & 0.99 & 200 & 200 \\
\hline \hline
\end{tabular}

3 repetições e para cada repetição o algoritmo genético foi executado 10 vezes, retornando a resposta média. Os valores encontrados podem ser observados na Tabela 2.

TABLE 2: VALOR DE fitness PARA DIFERENTES AJUSTES DE PARÂMETROS DO AG

\begin{tabular}{cccccc}
\hline \hline ponto & pCros & pMut & sPop & nGen & fitness \\
\hline \hline 1 & 0.01 & 0.01 & 10 & 10 & 14530.9 \\
\hline 2 & 0.01 & 0.01 & 10 & 105 & 14514.9 \\
\hline 3 & 0.01 & 0.01 & 10 & 200 & 14491.5 \\
\hline 4 & 0.01 & 0.01 & 105 & 10 & 14045.6 \\
\hline 5 & 0.01 & 0.01 & 105 & 105 & 14046.5 \\
\hline 6 & 0.01 & 0.01 & 105 & 200 & 14039.6 \\
\hline 7 & 0.01 & 0.01 & 200 & 10 & 13874.72 \\
\hline 8 & 0.01 & 0.01 & 200 & 105 & 13884.2 \\
\hline 9 & 0.01 & 0.01 & 200 & 200 & 13897.6 \\
\hline 10 & 0.01 & 0.5 & 10 & 10 & 14581.6 \\
\hline 11 & 0.01 & 0.5 & 10 & 105 & 14387.1 \\
\hline 12 & 0.01 & 0.5 & 10 & 200 & 14496.5 \\
\hline 13 & 0.01 & 0.5 & 105 & 10 & 14089.0 \\
\hline 14 & 0.01 & 0.5 & 105 & 105 & 14023.7 \\
\hline 15 & 0.01 & 0.5 & 105 & 200 & 14051.5 \\
\hline 16 & 0.01 & 0.5 & 200 & 10 & 13937.5 \\
\hline 17 & 0.01 & 0.5 & 200 & 105 & 13957.8 \\
\hline 18 & 0.01 & 0.5 & 200 & 200 & 13925.5 \\
\hline 19 & 0.01 & 0.99 & 10 & 10 & 14538.6 \\
\hline 20 & 0.01 & 0.99 & 10 & 105 & 14447.3 \\
\hline 21 & 0.01 & 0.99 & 10 & 200 & 14548.1 \\
\hline 22 & 0.01 & 0.99 & 105 & 10 & 14097.0 \\
\hline 23 & 0.01 & 0.99 & 105 & 105 & 14040.8 \\
\hline 24 & 0.01 & 0.99 & 105 & 200 & 14050.5 \\
\hline 25 & 0.01 & 0.99 & 200 & 10 & 13918.7 \\
\hline 26 & 0.01 & 0.99 & 200 & 105 & 13950.9 \\
\hline 27 & 0.01 & 0.99 & 200 & 200 & 13941.3 \\
\hline 28 & 0.5 & 0.01 & 10 & 10 & 14375.9 \\
\hline 29 & 0.5 & 0.01 & 10 & 105 & 14134.8 \\
\hline 30 & 0.5 & 0.01 & 10 & 200 & 14235.5 \\
\hline 31 & 0.5 & 0.01 & 105 & 10 & 13641.1 \\
\hline 32 & 0.5 & 0.01 & 105 & 105 & 13644.1 \\
\hline 33 & 0.5 & 0.01 & 105 & 200 & 13570.2 \\
\hline 34 & 0.5 & 0.01 & 200 & 10 & 13609.0 \\
\hline 36 & 0.5 & 0.01 & 200 & 105 & 13417.7 \\
\hline 37 & 0.5 & 0.01 & 200 & 200 & 13426.0 \\
\hline 39 & 0.5 & 0.5 & 10 & 105 & 14554.0 \\
\hline 31 & 0.5 & 0.5 & 10 & 200 & 14503.7 \\
\hline 3 & 0.5 & 105 & 10 & 13885.1 \\
\hline 36 & 0.5 & 105 & 105 & 13851.6
\end{tabular}

\begin{tabular}{cccccc}
\hline 42 & 0.5 & 0.5 & 105 & 200 & 13886.9 \\
\hline 43 & 0.5 & 0.5 & 200 & 10 & 13730.6 \\
\hline 44 & 0.5 & 0.5 & 200 & 105 & 13763.2 \\
\hline 45 & 0.5 & 0.5 & 200 & 200 & 13820.1 \\
\hline 46 & 0.5 & 0.99 & 10 & 10 & 14456.4 \\
\hline 47 & 0.5 & 0.99 & 10 & 105 & 14460.0 \\
\hline 48 & 0.5 & 0.99 & 10 & 200 & 14486.2 \\
\hline 49 & 0.5 & 0.99 & 105 & 10 & 13787.9 \\
\hline 50 & 0.5 & 0.99 & 105 & 105 & 13890.6 \\
\hline 51 & 0.5 & 0.99 & 105 & 200 & 13926.5 \\
\hline 52 & 0.5 & 0.99 & 200 & 10 & 13801.2 \\
\hline 53 & 0.5 & 0.99 & 200 & 105 & 13844.6 \\
\hline 54 & 0.5 & 0.99 & 200 & 200 & 13787.7 \\
\hline 55 & 0.99 & 0.01 & 10 & 10 & 14135.3 \\
\hline 56 & 0.99 & 0.01 & 10 & 105 & 14309.5 \\
\hline 57 & 0.99 & 0.01 & 10 & 200 & 14275.4 \\
\hline 58 & 0.99 & 0.01 & 105 & 10 & 13675.3 \\
\hline 59 & 0.99 & 0.01 & 105 & 105 & 13469.5 \\
\hline 60 & 0.99 & 0.01 & 105 & 200 & 13527.2 \\
\hline 61 & 0.99 & 0.01 & 200 & 10 & 13532.2 \\
\hline 62 & 0.99 & 0.01 & 200 & 105 & 13332.5 \\
\hline 63 & 0.99 & 0.01 & 200 & 200 & 13322.0 \\
\hline 64 & 0.99 & 0.5 & 10 & 10 & 14351.8 \\
\hline 65 & 0.99 & 0.5 & 10 & 105 & 14458.8 \\
\hline 66 & 0.99 & 0.5 & 10 & 200 & 14415.6 \\
\hline 67 & 0.99 & 0.5 & 105 & 10 & 13872.5 \\
\hline 68 & 0.99 & 0.5 & 105 & 105 & 13790.7 \\
\hline 69 & 0.99 & 0.5 & 105 & 200 & 13756.7 \\
\hline 70 & 0.99 & 0.5 & 200 & 10 & 13663.3 \\
\hline 71 & 0.99 & 0.5 & 200 & 105 & 13681.0 \\
\hline 72 & 0.99 & 0.5 & 200 & 200 & 13581.2 \\
\hline 73 & 0.99 & 0.99 & 10 & 10 & 14336.7 \\
\hline 74 & 0.99 & 0.99 & 10 & 105 & 14346.1 \\
\hline 75 & 0.99 & 0.99 & 10 & 200 & 14389.2 \\
\hline 76 & 0.99 & 0.99 & 105 & 10 & 13843.0 \\
\hline 77 & 0.99 & 0.99 & 105 & 105 & 13820.1 \\
\hline 78 & 0.99 & 0.99 & 105 & 200 & 13817.9 \\
\hline 79 & 0.99 & 0.99 & 200 & 10 & 13632.4 \\
\hline 80 & 0.99 & 0.99 & 200 & 105 & 13695.3 \\
\hline 81 & 0.99 & 0.99 & 200 & 200 & 13670.6 \\
\hline \hline & & & & & \\
\hline
\end{tabular}

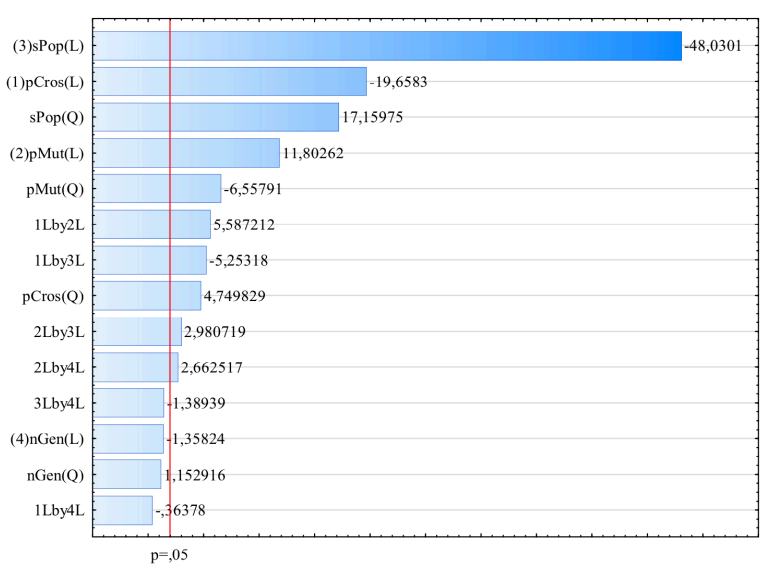

Fig. 4: Diagrama de Pareto. 


\section{RESULTADOS E DISCUSSÕES}

O diagrama de Pareto apresentado na Figura 4 mostra que os parâmetros que mais influenciaram na minimização do fitness são o tamanho da população ( $s$ Pop) e o percentual de crossover ( $p C r o s)$ quando avaliados separadamente. No entanto, quando combinados os parâmetros, o pCros $x$ pMut associado ao pCros $x$ sPop exerceram maior influência sob o fitness.

A relação entre os valores preditos e os valores ajustados (Figura 5) mostram alta correlação entre as variáveis estudadas. Não obstante, destaca-se, ainda, que o valor do coeficiente de determinação encontrado para os modelos estudados foi de $93,48 \%$, fato esse que, conforme descreve [10], indica elevada confiabilidade do modelo, uma vez o $R^{2}$ é o valor que mede o efeito da variável independente na variação dependente e, quanto mais próximo de 1 , maior a correlação.

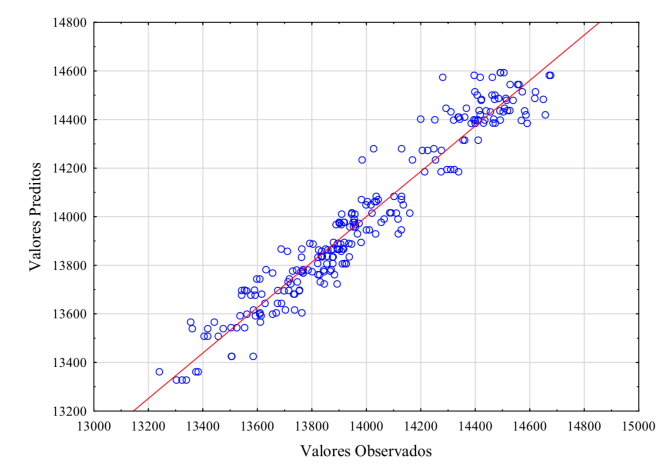

Fig. 5: Valores observados x Valores preditos.

Quando associadas, as variáveis $n G e n$ e $s P o p$ (Figura 6), observa-se que, independente dos valores de $n G e n$, quanto maior forem os valores de $s P o p$ melhor será a resposta, corroborando com o descrito no diagrama de pareto (Figura 4) que apresenta uma correlação direta entre a resposta e a variável sPop, ou seja, a medida que uma aumenta a outra possivelmente seguirá um comportamento semelhante.

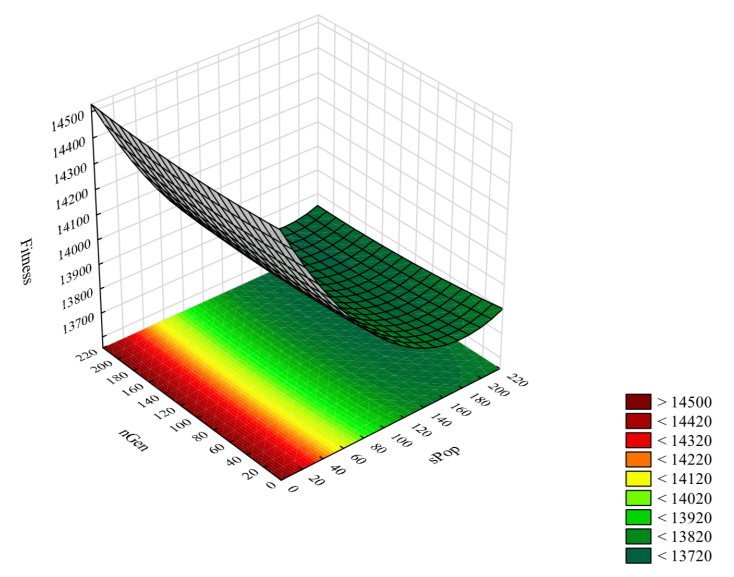

Fig. 6: Superfície de Resposta para a Interação dos parâmetros nGen x sPop

O valor de fitness observado durante a interação entre $s P o p$ x pMut (Figura 7) indica que as melhores condições de ação dessas variáveis são em faixas elevadas de sPop e reduzidas de pMut. Nesse sentido o diagrama de Pareto (Figura 4) também destaca a relação inversa entre a variável pMut e a resposta fitness, uma vez que o índice negativo indica que a redução de uma implicará no aumento da outra.

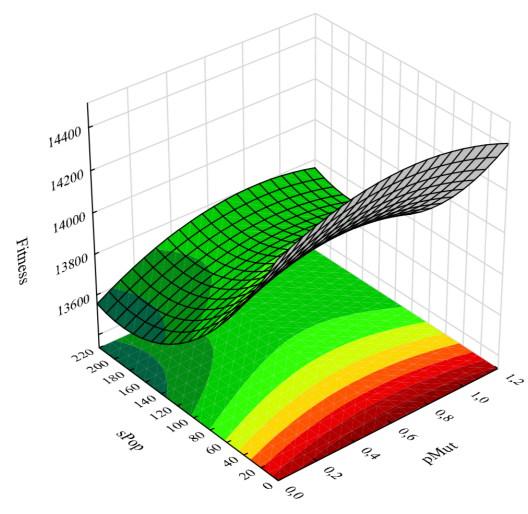

Fig. 7: Superfície de Resposta para a Interação dos parâmetros nMut x sPop.

Ao correlacionar as variáveis nGen e pMut (Figura 8) destaca-se que o $n$ Gen não exerce qualquer influência sobre a resposta, corroborando com o apresentado pelo Diagrama de Pareto (Figura 8). Por outro lado, a pMut apresentou o mesmo comportamento observado em outras interações das quais participou, ou seja, sua faixa desejável de atuação está em valores próximos ao parâmetro mínimo estudado $(-1)$.
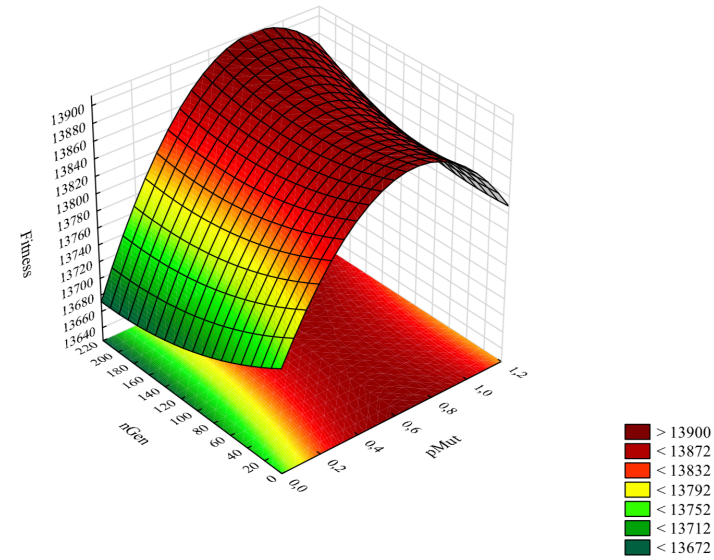

Fig. 8: Superfície de Resposta para a Interação dos parâmetros nGen $\mathrm{x}$ pMut.

No âmbito da correlação entre a $s P o p$ e a $p C r o s$ (Figura 9) observa-se que a melhor faixa da variável resposta encontrase em valores próximos ao parâmetro máximo (1) para ambas as variáveis independentes, confirmando, novamente, o comportamento descrito pelo Diagrama de Pareto (Figura 4). 


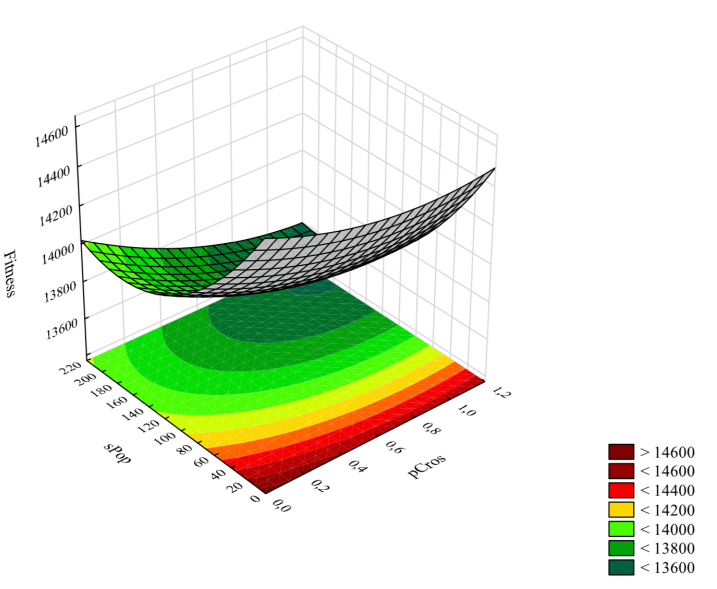

Fig. 9: Superfície de Resposta para a Interação dos parâmetros nCros x sPop.

\section{Conclusões}

Este trabalho apresentou a utilização de Algoritmos Genéticos (AG) para a área de Esteganografia, mostrando a aplicação da metodologia de Superfície de Resposta (MRS) como método de otimização de seus hiper-parâmetros. Um experimento foi conduzido de maneira a observar quatro hiperparâmetros do AG em questão. O experimento levou a concluir que a variável independente $n$ Gen não exerce influência significativa na variável resposta e que as melhores faixas de fitness encontram-se próximas ao parâmetro mínimo de $p M u t$ e máximo de sPop e $p$ Cros.

Neste sentido, conclui-se que após o uso da MSR, evitouse, tanto quanto possível, os riscos e erros associados ao método de tentativa e erro na definição de parâmetros do AG avaliado. Como observado nos gráficos na seção IV, a MSR indicou a região de valores para melhor configuração de parâmetros que levaram a minimização do valor da função fitness em questão.

\section{REFERENCES}

[1] F. Y. Shih, Digital Watermarking and Steganography: Fundamentals and Techniques, 1 st ed. Boca Raton, FL, USA: CRC Press, Inc., 2007

[2] G. Kipper, Investigator's Guide to Steganography. CRC Press, 2003.

[3] S. Katzenbeisser and F. Petitcolas, Information Hiding Techniques for Steganography and Digital Watermarking, ser. Artech House computer security series. Artech House, 2000.

[4] S. Mungmode, R. Sedamkar, and N. Kulkarni, "A modified high frequency adaptive security approach using steganography for region selection based on threshold value," Procedia Computer Science, vol. 79, pp. $912-921,2016$

[5] Gangeshawar and J. Attri, "Optimizing image steganography using genetic algorithm," International Journal of Engineering Trends and Technology (IJETT), vol. 24, no. 1, pp. 32-38, 2015.

[6] D. Montgomery, Design and Analysis of Experiments, 6th Edition Set. John Wiley \& Sons, Limited, 2007.

[7] M. Mitchell, An Introduction to Genetic Algorithms, 1st ed., ser. Complex Adaptive Systems. The MIT Press, 1996.

[8] O. Kramer, Genetic Algorithm Essentials, ser. Studies in Computational Intelligence. Springer, 2017, vol. 679.

[9] T.-Y. Wang and K.-B. Wu, "A parameter set design procedure for the simulated annealing algorithm under the computational time constraint," Computers \& Operations Research, vol. 26, no. 7, p. 665-678, Jun. 1999.
[10] P. R. M. de Azevedo, Introdução à estatística, 3rd ed. EDUFRN, 2016 